\title{
Correlation of prostate tumor eccentricity and Gleason scoring from prostatectomy and multi-parametric-magnetic resonance imaging
}

\author{
Rulon Mayer ${ }^{1,2}$, Charles B. Simone $\mathrm{II}^{3}$, Baris Turkbey ${ }^{4}$, Peter Choyke ${ }^{4}$ \\ ${ }^{1}$ University of Pennsylvania, Philadelphia, PA, USA; ${ }^{2}$ OncoScore, Garrett Park, MD, USA; ${ }^{3}$ New York Proton Center, New York, NY, USA; \\ ${ }^{4}$ National Institutes of Health, Bethesda, MD, USA
}

Contributions: (I) Conception and design: R Mayer; (II) Administrative support: R Mayer, CB Simone 2nd, P Choyke; (III) Provision of study materials or patients: B Turkbey, P Choyke; (IV) Collection and assembly of data: R Mayer; (V) Data analysis and interpretation: R Mayer; (VI) Manuscript writing: All authors; (VII) Final approval of manuscript: All authors.

Correspondence to: Rulon Mayer, PhD. University of Pennsylvania, Philadelphia, PA 19104, USA; OncoScore, Garrett Park, MD 20896, USA.

Email: mayerru@yahoo.com.

Background: Proliferating cancer cells interacting with their microenvironment affects a tumor's spatial shape. Elongation or roundness (eccentricity) of lung, skin, and breast cancers indicates the cancer's relative aggressiveness. Non-invasive determination of the prostate tumor's shape should provide meaningful input for prognostication and clinical management. There are currently few studies of prostate tumor shape, therefore this study examines the relationship between a prostate tumor's eccentricity, derived from spatially registered multi-parametric MRI and histology slides, and Gleason scores.

Methods: A total of 26 consecutive patients were enrolled in the study. Median patient age was 60 years (range, 49 to 75 years), median PSA was $5.8 \mathrm{ng} / \mathrm{mL}$ (range, 2.3 to $23.7 \mathrm{ng} / \mathrm{mL}$, and median Gleason score was 7 (range, 6 to 9). Multi-parametric MRI (T1, T2, Diffusion, Dynamic Contrast Enhanced) were resampled, rescaled, translated, and stitched to form spatially registered multi-parametric cubes. Multiparametric signatures that characterize prostate tumors were inserted into a target detection algorithm (Adaptive Cosine Estimator, ACE). Various detection thresholds were applied to discriminate tumor from normal tissue. Also, tumor shape was computed from the histology slides. Blobbing, labeling, and calculation of eccentricity using moments of inertia were applied to the multi-parametric MRI and histology slides. The eccentricity measurements were compared to the Gleason scores from 25 patients.

Results: From histology slides analysis: the correlation coefficient between the eccentricity for the largest blob and a weighted average eccentricity against the Gleason score ranged from -0.67 to -0.78 for all 18 patients whose tumor volume exceeded $1.0 \mathrm{cc}$. From multi-parametric MRI analysis: the correlation coefficient between the eccentricity for the largest blob for varying thresholds against the Gleason score ranged from -0.60 to -0.66 for all 25 patients showing contrast uptake in the Dynamic Contrast Enhancement (DCE) MRI.

Conclusions: Spherical shape prostate adenocarcinoma shows a propensity for higher Gleason score. This novel finding follows lung and breast adenocarcinomas but depart from other primary tumor types. Analysis of multi-parametric MRI can non-invasively determine the prostate tumor's morphology and add critical information for prognostication and disease management. Eccentricity of smaller tumors $(<1.0 \mathrm{cc})$ from MP-MRI correlates well with Gleason score, unlike eccentricity measured using histology of wholemount prostatectomy. 


\begin{abstract}
Keywords: Tumor morphology; supervised target detection; prostate cancer; multi-parametric MRI; histology of wholemount prostatectomy
\end{abstract}

Submitted Jan 12, 2021. Accepted for publication Apr 22, 2021.

doi: $10.21037 /$ qims-21-24

View this article at: http://dx.doi.org/10.21037/qims-21-24

\section{Introduction}

Staging components (1-3) such as intraprostatic tumor volumes/locations, metastatic extent, and lymph node involvement may indicate prostate tumor aggressiveness and provide important input for disease management. Tumor shape, whether spherical or elongated into an ellipsoid and quantified by a metric called eccentricity, is not usually measured and currently is not considered in staging for prostate cancer unlike breast $(4,5)$, lung $(6,7)$, and skin $(8,9)$ cancers. Due to the relative absence of studies regarding tumor eccentricity in prostate cancer, definitive understanding of the relationship between tumor shape and potential cancer progression is limited and its contribution for assessing the prognosis of a patient is uncertain to date.

Prior investigations (10-12) reported that the interaction between the proliferating cancer cells and their microenvironment determines a tumor's spatial shape. In contrast, it is hypothesized $(6,7)$ that formation of spherical aggressive lung adenocarcinomas is due to the inhibition of their growth by vessels, the bronchus, lymphatic tissues or the immune system in the normal lungs. To support understanding the generation of tumor shape, mathematical modeling of the spatial tumor growth may explain the process of longitudinal tumor growth $(13,14)$. Modeling might reveal some novel features of tumor growth. However, modeling is constrained by its inability to incorporate other clinical characteristics of tumors.

Cancer staging and scoring through invasive biopsies suffer from a number of deficiencies. Biopsies may lead to significant inter-observer variability and/or potential for sampling error leading to false negatives or underestimating the severity of the disease (or "underscoring"). Gleason score (GS) from needle biopsy can often differ from that determined with radical prostatectomy (RP) $(15,16)$ and with immediate repeat biopsies (17). Invasive biopsies can also inflict pain and result in possible complications such as hemorrhage and infection. Additional accurate diagnostic methods to establish the diagnosis are needed for patients with high persistent prostate specific antigen levels (PSA) but negative biopsies. For patients with low grade, low volume
PCa who are undergoing active surveillance and monitored for potential relapse or recurrence of $\mathrm{PCa}$ following therapy, MRI may provide valuable information. Moreover, the skill and experience of the reader may determine the quality of MRI interpretations. For PI-RADS (18), for example, the tumor aggressiveness evaluation requires experienced and specially trained radiologists.

\section{Methods}

\section{Overall description of histology and MP-MRI determination}

This study analyzed histology slides of patients who had undergone RP and were also imaged with multi-parametric MRI (19-23). The tumors were identified by experienced pathologists and radiologists on their respective imaging and the tumors were also assessed for clinical aggressiveness. The identified tumors were digitally outlined to create masks, blobs were autonomously identified from the masks, and moments of inertia computed to generate eccentricity for each blob. The largest blob's eccentricity and the weighted average eccentricity over all blobs were compared to the a priori defined Gleason score. In addition, Adaptive Cosine Estimator (ACE) (24-28) was applied to spatially registered the MRI $(27,28)$. After applying threshold to the ACE detection planes (28), blobs were labeled. The eccentricity for the largest blobs and a weighted average eccentricity were calculated and compared to the Gleason scores. The MP-MRI hypercubes and histology were constructed and processed from previously gathered data (19-23) but the analysis was totally independent of those earlier efforts.

This study followed two tracks (Figure 1) to determine the relationship between prostate tumor morphology (eccentricity) and Gleason score. One track examined tumors outlined on histology slides derived from wholemount prostatectomy as determined by a pathologist (denoted by solid blue arrows). The other track (denoted by solid red arrows) computed eccentricity from ACE applied to spatially registered multi-parametric MRI as determined 


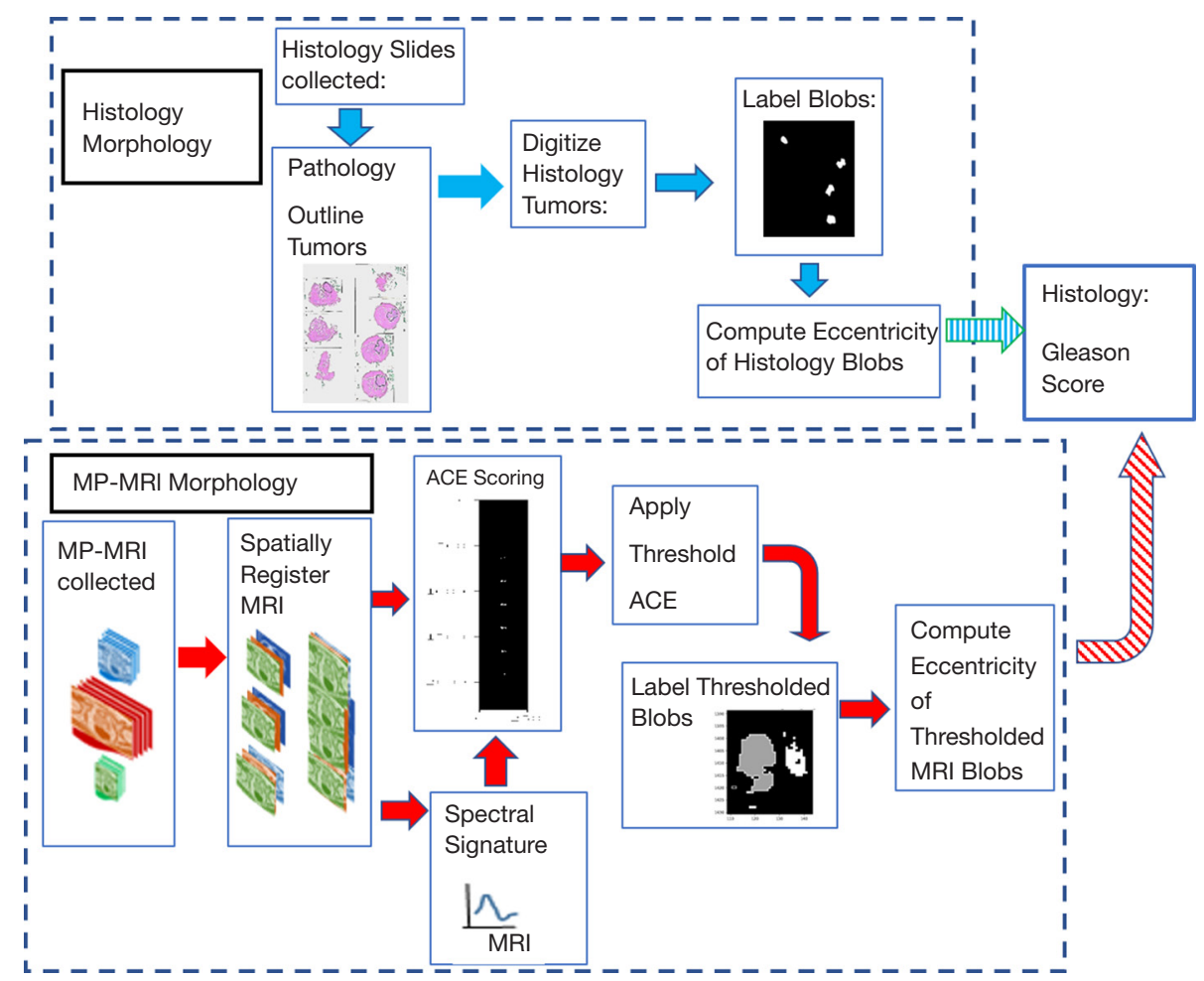

Figure 1 Schematic showing the overall procedure for generating tumor eccentricity from blobs derived from histology of wholemount prostatectomy specimens (solid blue arrows) and Adaptive Cosine Estimator (ACE) processing applied to multi-parametric MRI (MP-MRI). (solid red arrows) Blob eccentricity compared to Gleason scoring from histology (striped arrows).

by a radiologist. The analysis studied 25 of 26 patients who previously underwent RP, an analysis of the histology and Gleason score grading from pathologists, and scanned with MP-MRI. One of the 26 patients did not display contrast uptake in the MRI Dynamic Contrast Enhancement (DCE) images and was not analyzed. The calculated eccentricity, in both tracks, were derived from computing the moment of inertia for all pixels residing in a given blob. The eccentricity was then compared to the Gleason score of the tumor (denoted by striped arrows).

\section{Study design and population}

A portion of The Cancer Imaging Archive (TCIA) $(21,22)$ is a collection of National Institutes of Health (NIH) patient data from prostate MRI and histology from wholemount prostatectomy. The study was conducted in accordance with the Declaration of Helsinki (as revised in 2013). This retrospectively designed, single institution study was approved by the NIH Institutional Review Board, and was compliant with the Health Insurance
Portability and Accountability Act. Individual consent for this retrospective analysis was waived. Following previous protections implemented by TCIA, the data is publicly available and anonymized. This subsequent retrospective analysis of this data therefore did not require further review. From July 2008 to July 2009, 26 consecutive patients were enrolled in the study. Adenocarcinoma of the prostate was proved through biopsy in all patients. Table 1 summarizes the patient characteristics for this study. In this study, 18 patients had tumor size $>1 \mathrm{cc}$ and showed evidence of contrast uptake. Reference (28) describes the tumor volume in this data set in great detail. References (20-22) describe the patient characteristics. No restrictions were placed on tumor location within the prostate. This TCIA data set was chosen for pilot studies $(27,28)$ and for this current effort due to its easy accessibility, availability, and confirmed validity and soundness (19-22).

\section{Labeling and blob generation}

Blob detection methods are used in computer vision and 
Table 1 Patient summary

\begin{tabular}{lccc}
\hline & Median & Minimum & Maximum \\
\hline Patient age (years) & 60 & 49 & 75 \\
Gleason score & 7 & 6 & 9.39 \\
Tumor volume (cc) & 2 & 2.3 & 23.7 \\
PSA (ng/mL) & 5.8 & 3 & 180 \\
MRI to robotic prostatectomy time interval (days) & 60 & 3 \\
\hline
\end{tabular}

cc, cubic centimeters; PSA, prostate serum antigen.

apply to a digital image. Blob detection identifies areas that differ in properties within the image, such as brightness, compared to surrounding regions. A blob is a region in which all the points within a blob are considered to be similar, connected, and aggregated. Blobbing is based on whether the pixels form an 8-pixel connected neighborhood and bind them together into regions of interest. In this study, binary tumor masks are generated from histology slides and from images showing the pixels that exceed a threshold of ACE target detection applied to MP-MRI hypercubes. Specifically, the value of $1(0)$ or "True" ("False") is associated with tumor (background) in each masked image. Each "True" pixel peruses pixels within a given neighborhood (1 pixel away) to see if they are also "True" and are, therefore, connected, collected, and labeled as a member of a blob. Blobs smaller than $<0.1 \mathrm{cc}$ are filtered out.

\section{Eccentricity calculation}

Eccentricity and volume were computed for every labeled blob using custom software coded in Python 3. To compute the eccentricity, the largest axis $\mathrm{L}_{\mathrm{k}}$ and transverse moments $S_{k}$ are eigenvalues from the moment of inertia matrix I for the kth blob, where I

$$
I=\left\lceil\begin{array}{ll}
I_{x x} & I_{x y} \\
I_{y x} & I_{y y}
\end{array}\right]
$$

and the matrix components $\mathrm{I}_{\mathrm{xx}}, \mathrm{I}_{\mathrm{yy}}, \mathrm{I}_{\mathrm{xy}}$, and $\mathrm{I}_{\mathrm{yx}}$ are given by

$$
\begin{aligned}
I_{x x} & =\sum_{i=1}^{N}\left(y_{i}-y_{C M}\right)^{2}, \\
I_{y y} & =\sum_{i=1}^{N}\left(x_{i}-x_{C M}\right)^{2}, \\
I_{x y} & =I_{y x}=-\sum_{i=1}^{N}\left(x_{i}-x_{C M}\right)\left(y_{i}-y_{C M}\right)
\end{aligned}
$$

sum over $\mathrm{N}$ blob pixels where pixel i's horizontal $\left(\mathrm{x}_{\mathrm{i}}\right)$ and vertical $\left(\mathrm{y}_{\mathrm{i}}\right)$ position within the blob with a center of mass $(\mathrm{CM})$

$$
\begin{aligned}
& x_{C M}=\sum_{i=1}^{N}\left(x_{i}\right), \\
& y_{C M}=\sum_{i=1}^{N}\left(y_{i}\right)
\end{aligned}
$$

The eccentricity $E_{k}$ for the kth blob with a major axis $L_{k}$ and minor axis $S_{k}$ is given by

$$
E_{k}=\frac{L_{k}-S_{k}}{L_{k}}
$$

Eccentricity values $E_{k}$ range from 0 to 1 . A spherical shape has an eccentricity $E_{k}$ of 0 while a line has an eccentricity $E_{k}$ of 1 . Assuming density of unity, each Blob's volume $V_{k}$ is given by the image resolution $r$ and the number of pixels within each blob.

$$
V_{k}=r N=r \sum_{i=1}^{N}
$$

and $\mathrm{r}$ is given by

$$
r=r_{x} r_{y} r_{z}
$$

where $r_{z}$ is the slice thickness $(6 \mathrm{~mm})$ and $r_{x}$ and $r_{y}$ are the transverse or image resolution for each pixel, i.e. for MPMRI $r_{\mathrm{x}}=r_{\mathrm{y}}=1 \mathrm{~mm}$, for histology slices $\mathrm{r}_{\mathrm{x}}=\mathrm{r}_{\mathrm{y}}=0.0212 \mathrm{~mm}$ or 1,200 dots per inch.

The weighted eccentricity $W$ is a sum over B blobs, given by

$$
W=\frac{\sum_{k=1}^{B} V_{k} E_{k}}{\sum_{k=1}^{B} V_{k}}
$$



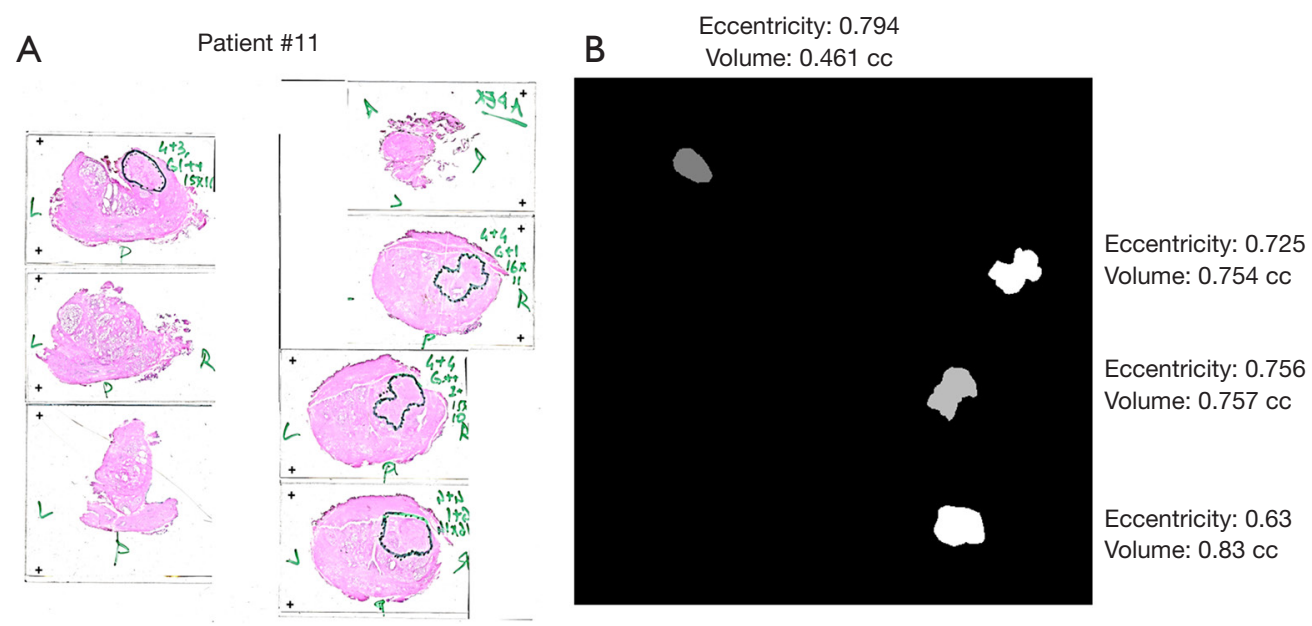

Figure 2 Schematic illustrating analysis of histology slices from wholemount prostatectomy. (A) shows a montage of 7 transverse slices of histology slides from a patient. A pathologist has marked the tumor. Each slice was stained with hematoxylin and eosin. The images were recorded with 1,200 dots per inch spatial resolution. The marked tumors are digitally traced. (B) displays the computed eccentricity and volume for the blobs denoted in (A).

\section{Whole mount prostatectomy and bistology}

Segmenting the prostate capsule on T2W MRI, and surface extracting from three-dimensional (3D) surfaces helps generate 3D models of each prostate $(19,20)$. Commercially available $3 \mathrm{D}$ computer software aided design of each mold. This design incorporated the deformation of the endorectal coil. A 3D printer (Dimension Elite 3D printer, Stratasys, Inc.) deposited styrene to fabricate each mold. After RP, the specimen was fixed in formalin at room temperature for 2 to 24 hours. This specimen was then placed in the customized $3 \mathrm{D}$ mold and sliced in axial $6 \mathrm{~mm}$ sections. Whole mount histopathology NIH patient specimens were sectioned in the customized mold for histopathology. Each slice was stained with hematoxylin and eosin. The images were recorded with 1,200 dots per inch spatial resolution. Two experienced pathologists, blinded to MRI, independently mapped for individual tumor foci, dimensions and Gleason scores. The sectioned molds corresponded to the axial plane of the MRI sections.

Figure 2 illustrates the determination of a tumor's morphology taken from histology slides from wholemount prostatectomy. Figure $2 A$ shows a montage of 7 transverse slices of histology slides take from a patient. A pathologist has marked the tumor. The marked tumors are digitally traced. Using Eqs. [1-6], the eccentricity and volume for the blobs denoted in Figure $2 A$ are shown in Figure $2 B$.

\section{Magnetic resonance imaging}

The MRI collection includes Diffusion Weighted Image, DCE, T1, and T2 images. Earlier studies $(19,20,23)$ described the pulse sequences. These studies $(19,20,23)$ combined an endorectal coil (BPX-30, Medrad) tuned to $127.8 \mathrm{MHz}$ and a 16 -channel cardiac coil (SENSE, Philips Medical Systems) on a 3T magnet (Achieva, Philips Medical Systems) without needing prior bowel preparation. Standard approaches were used to insert the endorectal coil. As part of the protocol, a prior study (23) defined triplanar T2W turbo spin echo, Diffusion Weighted MRI, 3DMR point resolved spectroscopy, axial pre-contrast T1-weighted axial 3D fast field echo, and DCE MRI sequences, and their detailed sequence parameters.

\section{Image processing, pre-analysis}

In the transverse direction, MRI images were digitally resampled and scaled $(27,28)$ to a common $1 \mathrm{~mm}$ resolution for all images. In the superior-inferior direction, the image set was resampled and resliced into $6 \mathrm{~mm}$ slices based on patient's table position. Additional minor translation and resampling helped spatially register the structural, diffusion, and DCE images at the pixel level. Additional adjustments may be needed due to the short time intervals between scans ( $<20$ minutes) and possible patient movement. The 


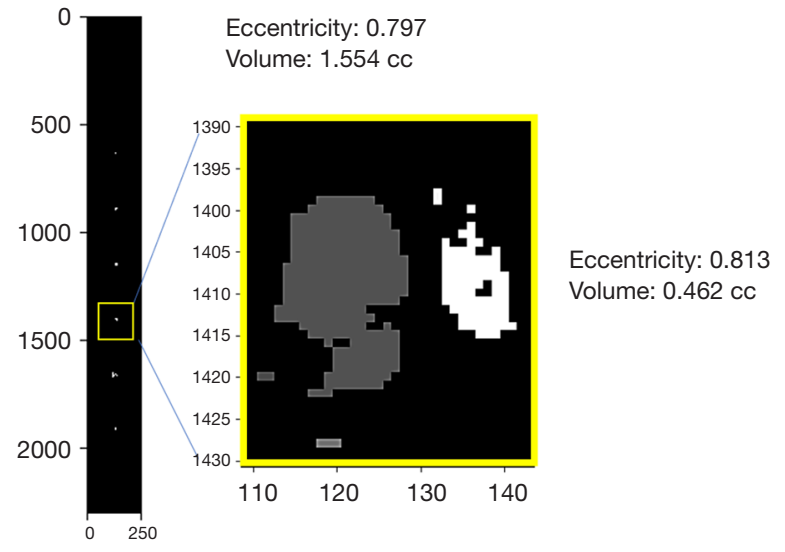

Figure 3 Schematic illustrating analysis of Adaptive Cosine Estimator (ACE) processing of Multi-parametric MRI (MP-MRI). Vertical and horizontal axis shows position within hypercube in pixels. (A) shows a mosaic of stitched ACE slices. Yellow rectangular outline shows the region that is magnified for display in (B). The computed eccentricity and volume for the expanded region in (A) and is shown in (B).

multiple axial cubes in three dimensions were "stitched" together into a narrow three-dimensional hypercube. Thus, a "four" dimensional (three-dimensional body volume plus the fourth dimension composed of MRI modalities) object is rearranged into three dimensions by stitching hypercubes.

By processing the DCE images, the tumor physiology helps distinguish lesions from normal tissues. The DCE is composed of a time series images. These images display the evolution of contrast material over several hundred seconds after contrast injection and follows the contrast uptake in the tissues. Often, prostate tumors are highly vascularized. Contrast material, through tumor vasculature, enters the tumor's extravascular space (but not the cells). Relative to the normal prostate organ, MRI contrast material quickly fills and empties prostate tumors. The tracer concentration in the tissue that supplies and empties through the tumor vasculature is described by a simple two-compartment model (27-30). This two-compartment model is used to create the washout or kep images, or rates for emptying and filling the tumor vasculature, respectively.

\section{$A C E$}

The multispectral Supervised Target Detection (STDA) methods (24-26), specifically the ACE \{Eq. [1] in reference (28)\}, were adapted for this medical application $(27,28)$. Multispectral
MRI data contain 7-components $(27,28)$ ( $\mathrm{T} 1$ (pre contrast), T1 (maximum contrast), T2, ADC, Diffusion Weighted Image-High $B\left(B=1,000 \mathrm{~s} / \mathrm{mm}^{2}\right)$, Washout or $\mathrm{k}_{\mathrm{ep}}$ from DCE) as described in Section $\mathrm{G}$. Each voxel $\mathrm{x}_{\mathrm{i}}$ is a 7-component vector, not the more common scaler. The supervised target detection algorithms use multispectral tumor signatures $\mathrm{S}$ that characterize the tumor (24-28) and to help detect the patient's tumor. $S$ the tumor signature, is the mean of $T$ target vector-voxels $x_{p}$ summed over $p$ target voxels data $\{\mathrm{Eq}$. $[8]\}$,

$$
S=\frac{1}{T} \sum_{p=1}^{T} x_{p}
$$

The signature $\mathrm{S}$ is inserted into the ACE algorithm to classify each pixel (26-28). ACE is the "whitened" (24-28) cosine between the test vector $\mathrm{x}_{\mathrm{i}}$ for pixel $\mathrm{i}$ and the target signature $\mathrm{S}$ vector $\{\mathrm{Eq}$. [8], above\} relative to $\mathrm{m}$ the background vector (normal prostate). $M$ is the mean value for each of the 7 component vectors [composed of DWI, T1, T2, ADC, DCE for this study, see References $(27,28)]$ of the delineated normal prostate. ACE is a conical hyperspace decision surface that determines whether a voxel is part of the background or normal prostate (large angle, small cosine) or target or tumor (small angle or large cosine) (24-28). The prostate image is manually outlined for all slices to help form an image mask. This mask restricts computations to the prostate volume in order to gather the background statistics [ $\mathrm{m}$ vector for the background or normal prostate tissue, see references $(27,28)]$, The specific selected ACE detection threshold segments the pixel into background or target. This threshold is set by the user based on previously examined data that optimized a desired feature such as correlation with a standard.

Figure 3 illustrates the determination of a tumor's morphology derived from spatially registered MP-MRI. Figure $3 A$ shows a mosaic of stitched ACE slices processed from spatially registered MP-MRI. Vertical and horizontal axis shows position within hypercube in pixels. Yellow rectangular outline shows the region that is magnified for display in Figure 3B. Using Eqs. [1-6], the eccentricity and volume for the expanded region in Figure $3 A$ are shown in Figure 3B.

\section{Results}

Figure 4 shows a scatterplot of the eccentricity from the largest blob and a weighted average $\{$ Eq. [7] $\}$ in all 18 patients with tumors sizes $>1.0 \mathrm{cc}$. The tumors were 


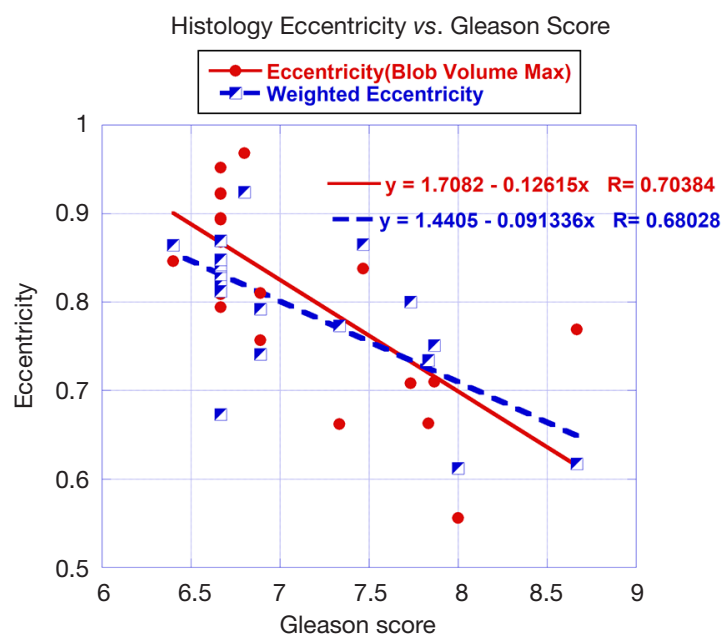

Figure 4 A scatterplot of the eccentricity from the largest blob (labeled as Blob Volume Max) in all 18 patients with tumor sizes $>1 \mathrm{cc}$ taken from analyzing the histology from wholemount prostatectomy plotted against the Gleason score.

from patients taken from analyzing the histology from wholemount prostatectomy. Figure 4 plots the eccentricity from the largest blob and a weighted (by mass) of all blobs \{Eq. [7]\} against the Gleason score derived from the pathologist's analysis of the histology. The correlation coefficient between the eccentricity for the largest blob and from the weighted eccentricity \{Eq. [7]\} from the histology against the Gleason score were -0.665 . and -0.783 , respectively with $\mathrm{P}$ values $<0.002$. The linearly fitted slope in both cases is negative, meaning that a spherical shape, rather than elongated, shows higher Gleason score and great aggressivity.

Figure 5 shows a scatterplot of the correlation coefficients resulting from fitting the eccentricity from the largest blob against the Gleason score derived from the pathologist's analysis of the histology in a range thresholds of ACE detection ranging from 0.35 to 0.65 in 0.05 increments for 25 patients, without a restriction on tumor size, but requires MRI contrast uptake. The correlation coefficient $\mathrm{R}$ between the eccentricity for the largest blob and a weighted average eccentricity against the Gleason score ranged from -0.60 to -0.68 for the ACE thresholds ( 0.35 to 0.55 ) achieving the highest $\mathrm{R}$ with $\mathrm{P}$ values $<0.003$. These 25 (of 26) patients also took up contrast material for the DCE portion of the MRI scan.

Figure 6 shows a scatterplot of the eccentricity from the largest blob and the weighted eccentricity $\{$ Eq. [7]\} of ACE

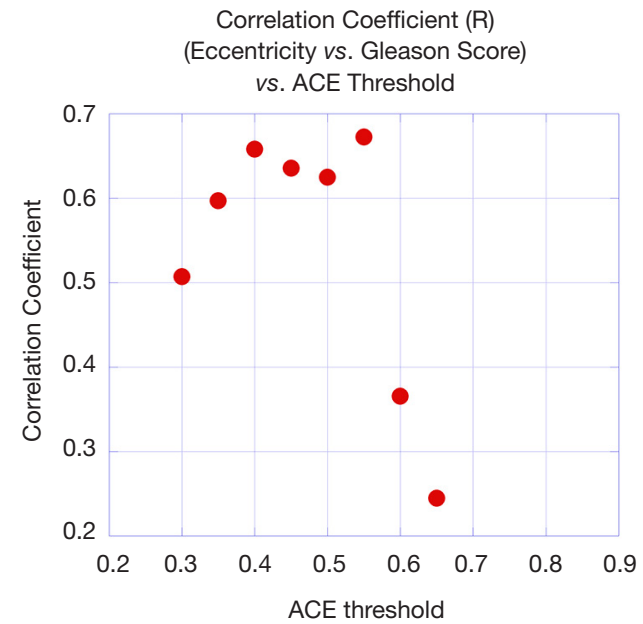

Figure 5 A scatterplot of the correlation coefficients resulting from fitting the eccentricity from the largest blob against the Gleason score derived from the pathologist's analysis of the histology $v s$. Adaptive Cosine Estimator (ACE) thresholds.

detection of thresholds ranging from 0.35 to 0.45 against the Gleason score derived from the pathologist's analysis of the histology in 25 patients. These 25 patients took up contrast material for the DCE portion of the MRI scan. The correlation coefficient between the eccentricity for the largest blob for varying thresholds against the Gleason score ranged from -0.60 to -0.65 with $\mathrm{P}$ values $<0.002$. The linearly fitted slope in both cases is negative, again meaning that a spherical shape, rather than elongated, shows higher Gleason score and greater aggressivity. However, comparing the weighted average for the eccentricity for MP-MRI to Gleason score achieved a poor correlation coefficient relative to using the eccentricity for the largest blob.

\section{Discussion}

This study demonstrates that both MP-MRI and histopathology shows that prostate tumors aggressiveness is correlated with tumor shape eccentricity. Specifically, this study determined that spherical, rather than elongated, tumor shape is indicative of higher Gleason score and greater tumor aggressiveness. Adenocarcinomas in the breast (5) and the lung $(6,7)$ also show greater aggressiveness when they appear spherical. Yoon (6) used CT to monitor lung adenocarcinomas and tumor doubling times and found a positive correlation between eccentricity and tumor doubling times. Baba (7) measured the geometry 


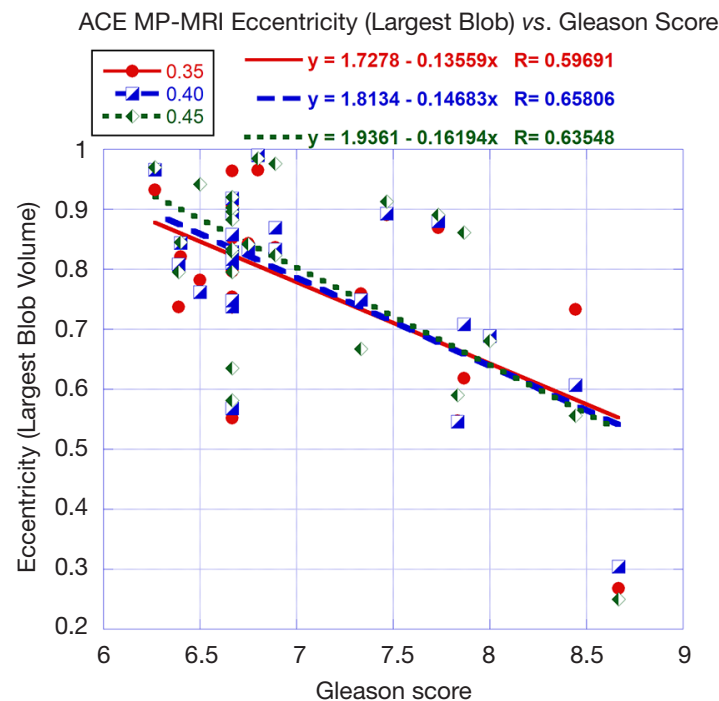

Figure 6 A scatterplot of the eccentricity from the largest blob of Adaptive Cosine Estimator (ACE) Multi-parametric MRI (MPMRI) for detection thresholds ranging from 0.35 to 0.45 in 25 patients against the Gleason score derived from the pathologist's analysis of the histology. Fitting parameters and correlation coefficients for linear fits of the eccentricity against Gleason score are also shown.

for resected lung adenocarcinomas and found longer survival for elongated relative to globular tumors. However, this is not always the case as breast cancer patients with negative hormone receptor and positive HER2 expression show greater aggressiveness when they are more elongated and asymmetric (4). Similarly, in malignant melanomas $(8,9)$, asymmetry in a tumor's shape may indicate its aggressiveness.

Unlike earlier work, this study utilized novel methods to compute the moments of inertia to find the eccentricity. All pixels were digitally incorporated into the calculation. More typically, the pathologist (or urologist) (6) visually inspected the tumors for smallest and largest axis. Another earlier eccentricity calculation computed the ratio of the inferred ellipsoid and sphere from excised biopsy and then approximated the tumor eccentricity (4). Using supervised target detection in MP-MRI to delineate the tumor further reduces the possible subjective assessment of a radiologist and pathologist. The method in this study is less subjective and, therefore, more readily replicated in other clinics.

This study found that among the 18 patients with the largest tumors, there was a negative correlation between Gleason scores and assessed eccentricity for pathology of histology slices. In contrast, 25 out of 26 patient studies were used in the assessments of MP-MRI. These findings suggest that smaller tumors may be affected during the wholemount prostatectomy/histology preparation process rendering less correlation using pathology assessment of the wholemount prostatectomy histology slices. Sectioning of tissues and preparation for histology slides can distort the tissues. Imaging a patient with MRI, rather than evaluating the tumor with histologic sectioning, might retain the relevant tissue shape in its natural state.

A number of metrics can characterize the eccentricity for prostate cancer. Only a weighted average over all blobs with the histology slide performed as well as the eccentricity for the largest blob in following Gleason score. However, and notably, comparing the weighted average for the eccentricity for MP-MRI to Gleason score achieved a poor correlation coefficient relative to using the eccentricity for the largest blob.

ACE values range from -1 to 1 (24-28) so using fixed thresholds is expected to generate robust criteria for selecting blobs. A fixed threshold (0.45) applied to ACE scoring achieved the highest correlation between eccentricity and Gleason scoring. Other threshold techniques, such as Otsu threshold (31) failed to achieve high correlation between any eccentricity measure and Gleason score.

While the current study had several novel findings, it also had several limitations. First, the methods used only assessed the tumor in two dimensions. Additional study is needed to determine if refinements of the technique provide better correlation. Additionally, while consecutive patients were utilized to minimize bias, an overall limited sample size was studied for this pilot analysis. Additional confirmatory studies are needed with greater patient numbers to determine if eccentricity may have a future role in prostate cancer staging and surveillance.

\section{Conclusions}

This is the first systematic study of prostate tumor shape and its connection to Gleason score and tumor aggressiveness. This study examined prostate tumors in spatially registered MP-MRI and pathology assessments of prostatectomy. A spherical, rather than elongated, prostate adenocarcinoma primary tumor shape, shows a propensity of higher Gleason score and greater potential aggressiveness. This behavior of the eccentricity for adenocarcinoma in the prostate follows the behavior of adenocarcinoma in the lung and breast but departs from other primary tumor types. MP-MRI can 
non-invasively determine the prostate tumor's morphology and provide additional, critical information for disease management. However, sectioning for histology slices may distort the shape of tumors, especially small tumors $(<1.0 \mathrm{cc})$ relative to in-vivo MRI.

Additional study is needed to determine the utility of adding eccentricity of prostate tumors to inform staging and prognosis of prostate cancer.

\section{Acknowledgments}

Funding: None.

\section{Footnote}

Conflicts of Interest: All authors have completed the ICMJE uniform disclosure form (available at http://dx.doi. org/10.21037/qims-21-24). Dr. PC reports that he has US government patents that generate some royalties that are distantly related to content (Fusion biopsy system for prostate MRI and AI systems for prostate MRI). However, these are not in conflict with the content of this article. The other authors have no conflicts of interest to declare.

Ethical Statement: The authors are accountable for all aspects of the work in ensuring that questions related to the accuracy or integrity of any part of the work are appropriately investigated and resolved. The study was conducted in accordance with the Declaration of Helsinki (as revised in 2013). This retrospectively designed, single institution study was approved by the NIH Institutional Review Board, and was compliant with the Health Insurance Portability and Accountability Act. And individual consent for this retrospective analysis was waived.

Open Access Statement: This is an Open Access article distributed in accordance with the Creative Commons Attribution-NonCommercial-NoDerivs 4.0 International License (CC BY-NC-ND 4.0), which permits the noncommercial replication and distribution of the article with the strict proviso that no changes or edits are made and the original work is properly cited (including links to both the formal publication through the relevant DOI and the license). See: https://creativecommons.org/licenses/by-nc-nd/4.0/.

\section{References}

1. Sobin L, Wittekind C. TNM classification of malignant tumors. 5th ed. New York: John Wiley and Sons, Inc, 1997.

2. McNeal JE. Cancer volume and site of origin of adenocarcinoma in the prostate: relationship to local and distant spread. Hum Pathol 1992;23:258-66.

3. Villers AA, McNeal JE, Redwine EA, Freiha FS, Stamey TA. Pathogenesis and biological significance of seminal vesicle invasion in prostatic adenocarcinoma. J Urol 1990;143:1183-7.

4. Moon HG, Kim N, Jeong S, Lee M, Moon H, Kim J, Yoo TK, Lee HB, Kim J, Noh DY, Han W. The Clinical Significance and Molecular Features of the Spatial Tumor Shapes in Breast Cancers. PLoS One 2015;10:e0143811.

5. Bae MS, Seo M, Kim KG, Park IA, Moon WK.

Quantitative MRI morphology of invasive breast cancer: correlation with immunohistochemical biomarkers and subtypes. Acta Radiol 2015;56:269-75.

6. Yoon HJ, Park H, Lee HY, Sohn I, Ahn J, Lee SH. Prediction of tumor doubling time of lung adenocarcinoma using radiomic margin characteristics. Thorac Cancer 2020;11:2600-9.

7. Baba T, Uramoto H, Takenaka M, Oka S, Shigematsu Y, Shimokawa H, Hanagiri T, Tanaka F. The tumour shape of lung adenocarcinoma is related to the postoperative prognosis. Interact Cardiovasc Thorac Surg 2012;15:73-6.

8. Daniel Jensen J, Elewski BE. The ABCDEF Rule: Combining the "ABCDE Rule" and the "Ugly Duckling Sign" in an Effort to Improve Patient Self-Screening Examinations. J Clin Aesthet Dermatol 2015;8:15.

9. Ali AH, Li J, Yang G. Automating the ABCD Rule for Melanoma Detection: A Survey. IEEE Access 2020;8:83333-46.

10. Baghban R, Roshangar L, Jahanban-Esfahlan R, Seidi K, Ebrahimi-Kalan A, Jaymand M, Kolahian S, Javaheri $\mathrm{T}$, Zare P. Tumor microenvironment complexity and therapeutic implications at a glance. Cell Commun Signal 2020;18:59.

11. Ungefroren H, Sebens S, Seidl D, Lehnert H, Hass R. Interaction of tumor cells with the microenvironment. Cell Commun Signal 2011;9:18.

12. Anderson AR, Weaver AM, Cummings PT, Quaranta $\mathrm{V}$. Tumor morphology and phenotypic evolution driven by selective pressure from the microenvironment. Cell 2006;127:905-15.

13. Szabó A, Merks RM. Cellular potts modeling of tumor growth, tumor invasion, and tumor evolution. Front Oncol 2013;3:87.

14. Bearer EL, Lowengrub JS, Frieboes HB, Chuang YL, Jin F, Wise SM, Ferrari M, Agus DB, Cristini V. Multiparameter 
computational modeling of tumor invasion. Cancer Res 2009;69:4493-501.

15. King CR, Long JP. Prostate biopsy grading errors: a sampling problem? Int J Cancer 2000;90:326-30.

16. Epstein JI, Feng Z, Trock BJ, Pierorazio PM. Upgrading and downgrading of prostate cancer from biopsy to radical prostatectomy: incidence and predictive factors using the modified Gleason grading system and factoring in tertiary grades. Eur Urol 2012;61:1019-24.

17. Berglund RK, Masterson TA, Vora KC, Eggener SE, Eastham JA, Guillonneau BD. Pathological upgrading and up staging with immediate repeat biopsy in patients eligible for active surveillance. J Urol 2008;180:1964-7; discussion 1967-8.

18. Weinreb JC, Barentsz JO, Choyke PL, Cornud F, Haider MA, Macura KJ, Margolis D, Schnall MD, Shtern F, Tempany CM, Thoeny HC, Verma S. PI-RADS Prostate Imaging - Reporting and Data System: 2015, Version 2. Eur Urol 2016;69:16-40.

19. Shah V, Pohida T, Turkbey B, Mani H, Merino M, Pinto PA, Choyke P, Bernardo M. A method for correlating in vivo prostate magnetic resonance imaging and histopathology using individualized magnetic resonancebased molds. Rev Sci Instrum 2009;80:104301.

20. Turkbey B, Mani H, Shah V, Rastinehad AR, Bernardo M, Pohida T, Pang Y, Daar D, Benjamin C, McKinney YL, Trivedi H, Chua C, Bratslavsky G, Shih JH, Linehan WM, Merino MJ, Choyke PL, Pinto PA. Multiparametric 3T prostate magnetic resonance imaging to detect cancer: histopathological correlation using prostatectomy specimens processed in customized magnetic resonance imaging based molds. J Urol 2011;186:1818-24.

21. Choyke P, Turkbey B, Pinto P, Merino M, Wood B, Data From PROSTATE-MRI. The Cancer Imaging Archive, 2016.

22. Clark K, Vendt B, Smith K, Freymann J, Kirby J, Koppel P, Moore S, Phillips S, Maffitt D, Pringle M, Tarbox L, Prior
F. The Cancer Imaging Archive (TCIA): maintaining and operating a public information repository. J Digit Imaging 2013;26:1045-57.

23. Turkbey B, Pinto PA, Mani H, Bernardo M, Pang Y, McKinney YL, Khurana K, Ravizzini GC, Albert PS, Merino MJ, Choyke PL. Prostate cancer: value of multiparametric MR imaging at $3 \mathrm{~T}$ for detection-histopathologic correlation. Radiology 2010;25 5:89-99.

24. Jain AK. Fundamentals of Digital Image Processing. Upper Saddle River, NJ: Prentice Hall, 1989.

25. Richards JA, Jia X, Remote Sensing Digital Image Analysis, New York: Springer-Verlag, 1999.

26. Manolakis D, Shaw G. Detection algorithms for hyperspectral imaging applications. IEEE Signal Process Mag 2002;19:29-43.

27. Mayer R, Simone CB 2nd, Skinner W, Turkbey B, Choykey P. Pilot study for supervised target detection applied to spatially registered multiparametric MRI in order to non-invasively score prostate cancer. Comput Biol Med 2018;94:65-73.

28. Mayer R, Simone CB 2nd, Turkbey B, Choyke P. Algorithms applied to spatially registered multi-parametric MRI for prostate tumor volume measurement. Quant Imaging Med Surg 2021;11:119-32.

29. Tofts PS, Brix G, Buckley DL, Evelhoch JL, Henderson E, Knopp MV, Larsson HB, Lee TY, Mayr NA, Parker GJ, Port RE, Taylor J, Weisskoff RM. Estimating kinetic parameters from dynamic contrast-enhanced T(1)-weighted MRI of a diffusable tracer: standardized quantities and symbols. J Magn Reson Imaging 1999;10:223-32.

30. Tofts PS. T1-weighted DCE Imaging Concepts: Modelling, Acquisition and Analysis. Magnetom Flash 2010;3:31-9.

31. Otsu N. A threshold selection method from gray-level histograms. IEEE Trans Sys Man Cyber 1979;9:62-6.
Cite this article as: Mayer R, Simone CB 2nd, Turkbey B, Choyke P. Correlation of prostate tumor eccentricity and Gleason scoring from prostatectomy and multi-parametricmagnetic resonance imaging. Quant Imaging Med Surg 2021;11(10):4235-4244. doi: 10.21037/qims-21-24 